\title{
Supplementary Information For: Controlling Proton Conductivity with Light: A Scheme based on Photoacid Doping of Materials
}

\author{
Shima Haghighat, Sarah Ostresh, and Jahan M. Dawlaty* \\ Department of Chemistry, University of Southern California \\ E-mail: dawlaty@usc.edu
}

KEYWORDS: Proton conductivity, photo-acid,

*To whom correspondence should be addressed 


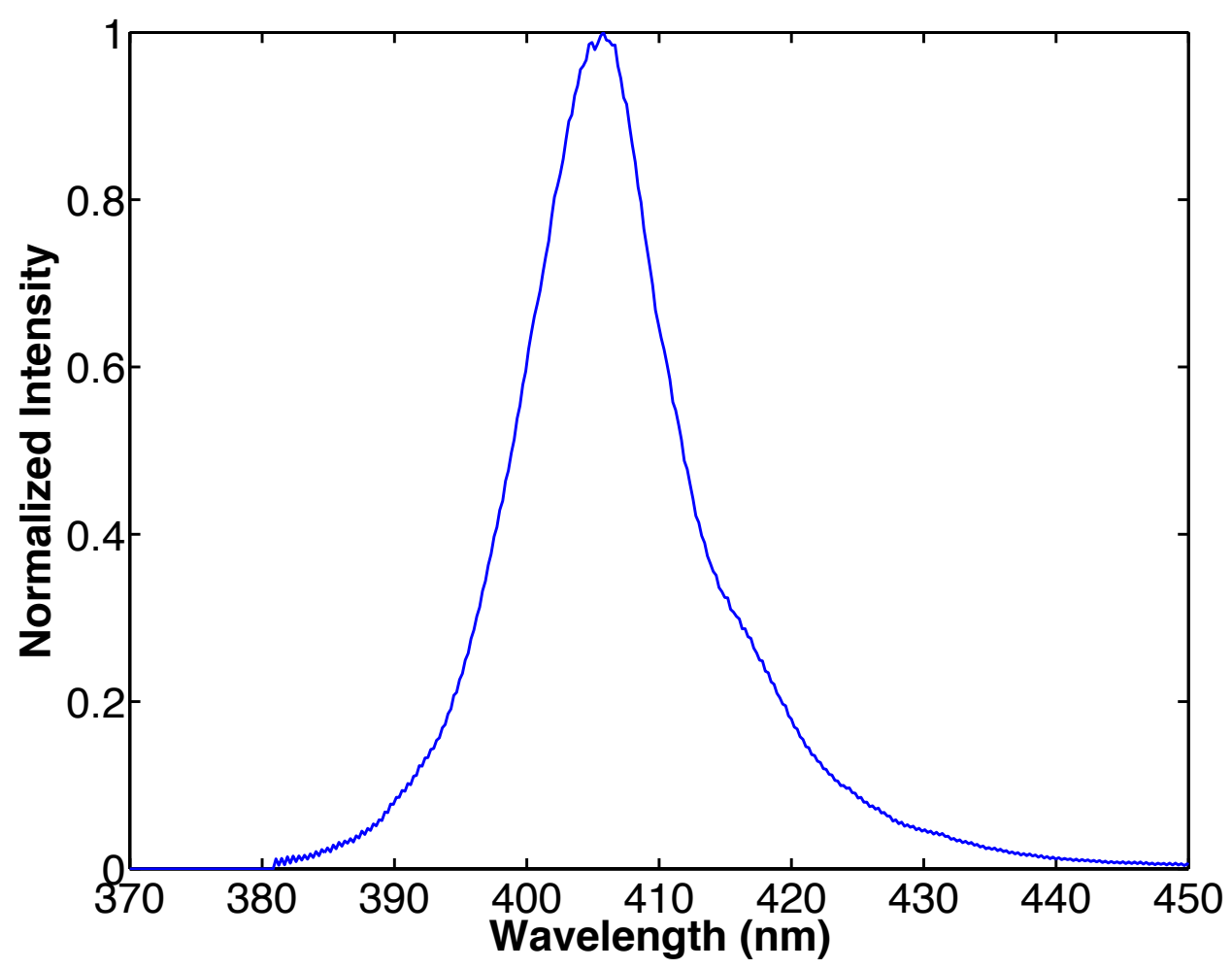

Figure 1: THORLABS 405 nm mounted LED lamp spectrum. 


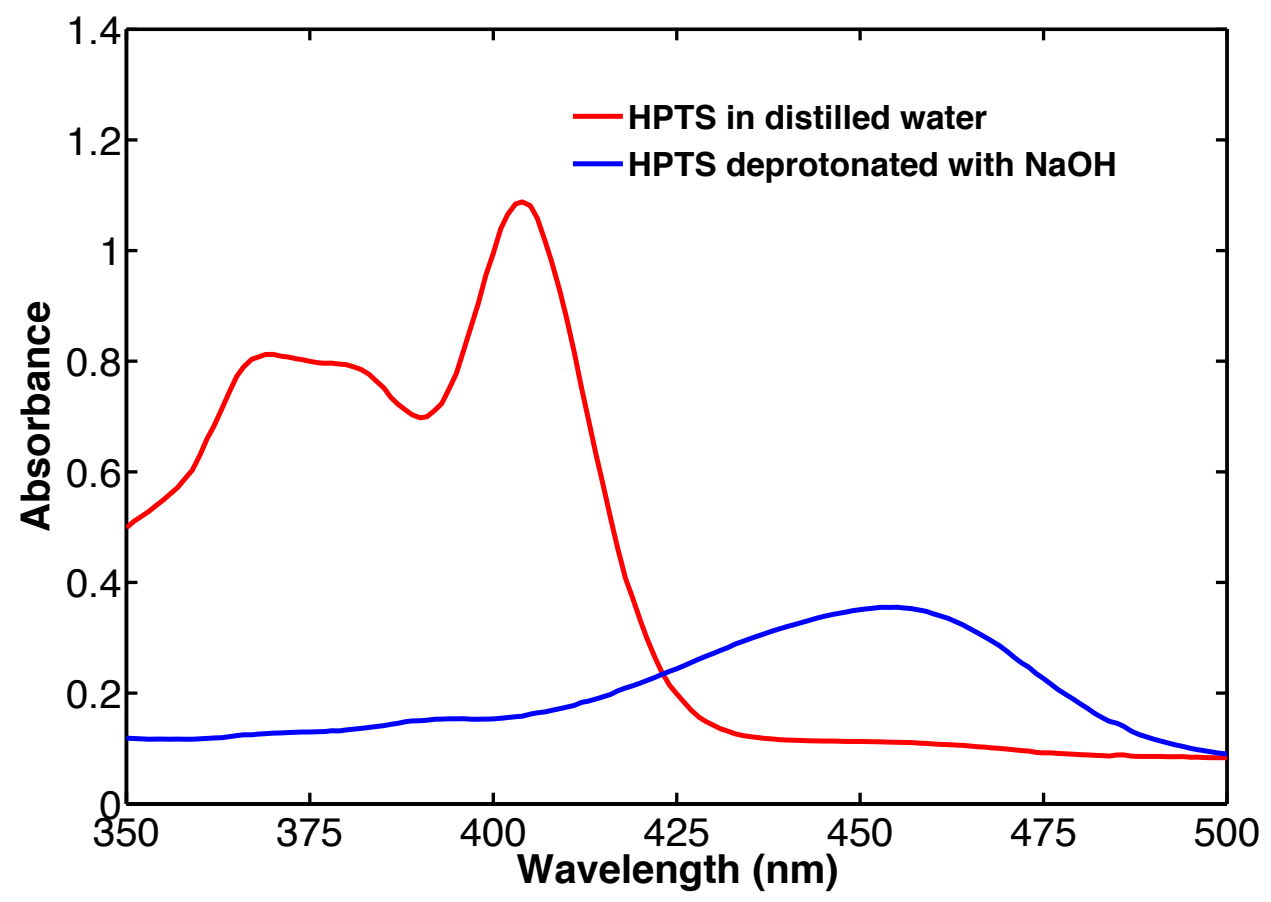

Figure 2: Absorption spectra of HPTS dissolve in distilled water (red spectrum) and after adding $\mathrm{NaOH}$ and deprotonation of HPTS (blue spectrum).

\section{Relation of the real part of impedance to the magnitude of the War- burg element}

In our experimental data the real part of the impedance of the circuit increases upon shining light. We have modeled and interpreted the response of the entire circuit to light and have arrived at the conclusion that the light illumination results into reduction of the the Warburg impedance as supported and justified in the main text. This may seem counterintuitive that reduction of the Warburg impedance would result into increase in the real part of the impedance of the circuit. However, it should be born in mind that $Z_{w}$ is complex, and when put in parallel in a circuit with a capacitor, it is possible that reduction of its magnitude would result into increase in the real part of the circuit impedance. In the following we prove this point.

Consider a Warburg element:

$$
Z_{w}=\frac{(1-i) A_{w}}{\sqrt{2 \omega}}
$$


in parallel with a capacitor:

$$
Z_{c}=\frac{1}{i \omega C}
$$

The overall impedance becomes:

$$
Z=\frac{1}{1 / Z_{w}+1 / Z_{c}}=\frac{1}{\frac{(1+i) \sqrt{\omega}}{\sqrt{2} A_{w}}+i c \omega}
$$

The real part of the above is:

$$
\operatorname{Re}(Z)=\frac{1}{\frac{\sqrt{2 \omega}\left(c^{2} \omega A_{w}^{2}+1\right)}{A_{w}}+2 c \omega}
$$

Next we take the derivative of the above with respect to $A_{w}$ :

$$
\frac{\partial \operatorname{Re}(Z)}{\partial A_{w}}=\frac{\sqrt{2}\left(1-c^{2} \omega A_{w}^{2}\right)}{\sqrt{\omega}\left(\sqrt{2} c^{2} \omega A_{w}^{2}+2 c \sqrt{\omega} A_{w}+\sqrt{2}\right)^{2}}
$$

The above can be negative when:

$$
A_{w}^{2}<\frac{1}{c^{2} \omega}
$$

When the Warburg impedance magnitude and its parallel capacitance satisfy the above relation a decrease in $A_{w}$ results into a increase in $\operatorname{Re}(Z)$. Thus justifying the point made at the beginning of this section. Including the resistance in series with $Z_{w}$ results into a more complicated equation for the conditions in which the reduction $A_{w}$ can increase $\operatorname{Re}(Z)$. However, it does not provide any further insight and is not included here. 


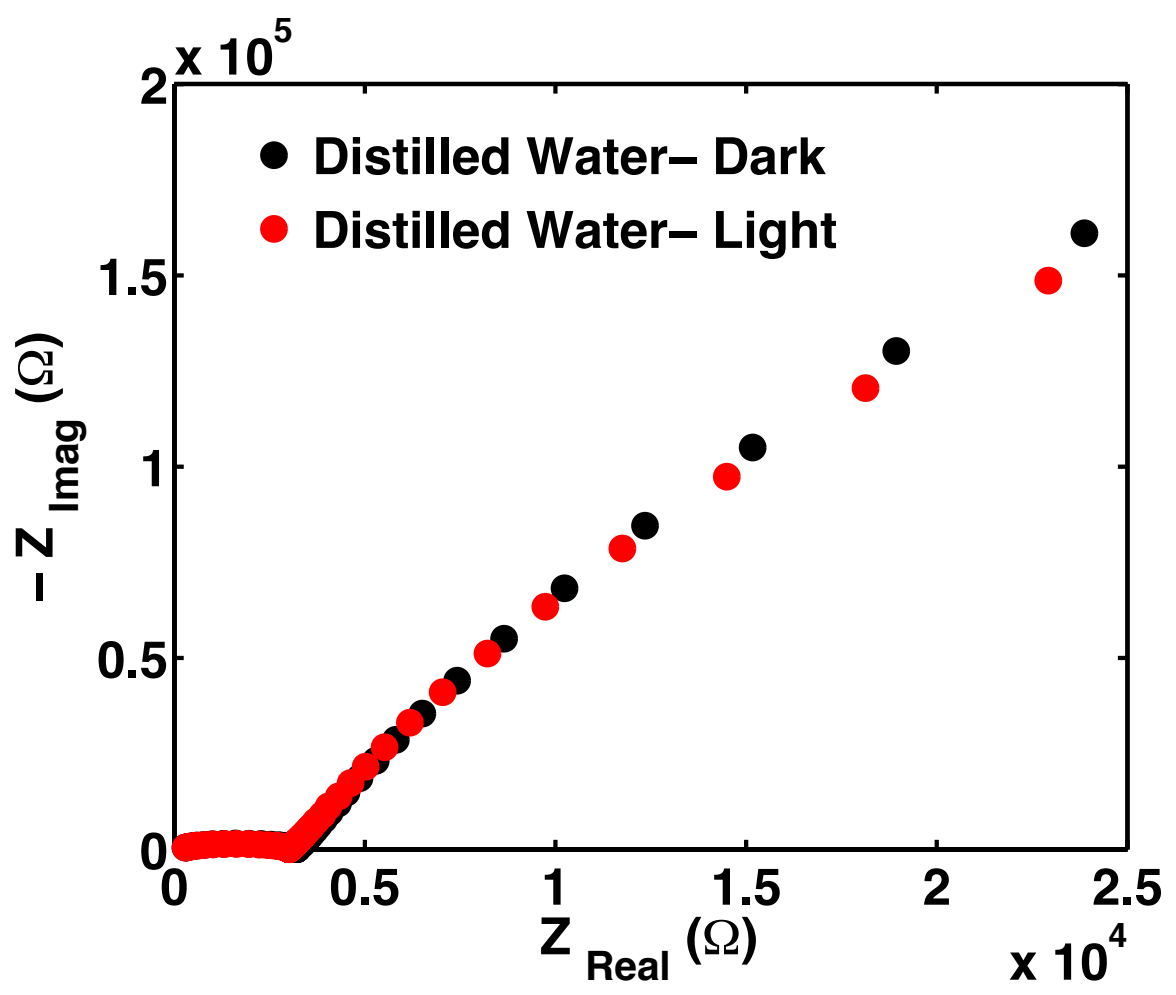

Figure 3: Nyquist plot of pure distilled water over the frequency range of $0.1 \mathrm{~Hz}$ to $1 \mathrm{MHz}$ in the presence and absence of light. 